\title{
MTHFR C677T polymorphism and osteoporotic fracture in postmenopausal women: a meta-analysis
}

\author{
J.Z. Guan, M. Wu, Y.Z. Xiao, J.S. Zhou and Z.D. Wang \\ Anhui Key Laboratory of Tissue Transplantation, \\ Institute of Orthopaedics \& Traumatology, \\ The First Affiliated Hospital of Bengbu Medical College, Bengbu, China \\ Corresponding author: J.S. Zhou \\ E-mail: zhoujs12399@yahoo.com.cn
}

Genet. Mol. Res. 13 (3): 7356-7364 (2014)

Received March 18, 2013

Accepted October 30, 2013

Published September 12, 2014

DOI http://dx.doi.org/10.4238/2014.September.12.1

\begin{abstract}
Numerous studies have evaluated the association between MTHFR C677T polymorphism and osteoporotic fracture risk in postmenopausal women. However, the results have been inconsistent. We performed a meta-analysis of the association between MTHFR C677T polymorphism and osteoporotic fracture risk in postmenopausal women. Only seven case-control studies were retrieved, with a total of 4258 patients and 3454 healthy controls. Meta-analysis results showed no significant association between MTHFR C677T polymorphism and osteoporotic fracture risk in postmenopausal women for all genetic models (for TT $v s \mathrm{CC}$ : OR = $0.99,95 \% \mathrm{CI}=0.72-1.39$; for $\mathrm{TT} v s \mathrm{TC}: \mathrm{OR}=1.02,95 \% \mathrm{CI}=0.87$ 1.20; for $\mathrm{CC}+\mathrm{TC}$ vs TT: $\mathrm{OR}=0.96,95 \% \mathrm{CI}=0.71-1.28$; for TT+TC vs $\mathrm{CC}: \mathrm{OR}=0.93,95 \% \mathrm{CI}=0.84-1.03)$. In the subgroup analysis by ethnicity, the results also showed no significant association between MTHFR C677T polymorphism and susceptibility to osteoporotic
\end{abstract}


fracture in postmenopausal women in both Caucasian and Asian populations. In conclusion, this meta-analysis suggests that MTHFR C677T polymorphism may not be associated with susceptibility to osteoporotic fracture in postmenopausal women.

Key words: C677T; Meta-analysis; Gene polymorphism; Postmenopausal women

\section{INTRODUCTION}

Osteoporosis is a common metabolic bone disorder characterized by reduced bone mass, increased skeletal fragility and microarchitectural deterioration, and eventually susceptibility to fractures, particularly of the spine, hip, wrist, humerus, and pelvis. Osteoporotic fracture is a major health care problem in the Western world. It is associated with impaired functioning, decreased quality of life, increased nursing home admission and increased mortality (Pongchaiyakul et al., 2005). Melton (1997) reported that approximately $30 \%$ of postmenopausal white women in the United States have osteoporosis, of whom $25 \%$ have a vertebral fracture, imposing a major economic burden on society. There are some factors that may potentially influence osteoporotic fracture: older age ( $>75$ years), low heel quantitative ultrasound stiffness index $(<78 \%)$, history of fracture, recent fall, and a failed chair test. In addition, genetic epidemiological studies of osteoporotic fractures have provided direct evidence supporting the concept that susceptibility to osteoporotic fractures is under genetic control, with estimated heritability ranging from 0.2 to 0.68 (Michaelsson et al., 2005).

Homocysteine is a naturally occurring amino acid found in blood plasma. It has been suggested that total blood homocysteine concentration is associated with the risk of ischemic heart disease and stroke. Recently, several studies have indicated that high homocysteine levels are involved in the pathology of osteoporosis fractures (van Meurs et al., 2004; McLean et al., 2004). The underlying pathophysiological mechanism is not completely understood. A potential mechanism is that homocysteine specifically interferes with the formation of collagen cross-links (Jackson, 1973). Collagen cross-links are important for the stability and strength of the collagen network, and interference in the formation of cross-links results in an altered bone matrix, which then results in fragile bone (Lubec et al., 1996).

5,10-methylenetetrahydrofolate reductase (MTHFR) catalyzes the reduction of 5-methylenetetrahydrofolate to 5-methyltetrahydrofolate, the predominant circulatory form of folate and the methyl group donor required for the remethylation of homocysteine to methionine. Reduced MTHFR activity has been reported to lead to increased homocysteine levels (Frosst et al., 1995). One common allelic polymorphism (C677T) in the MTHFR gene has been shown to lead to reduced MTHFR activity and thereby increased risk of hyperhomocysteinemia. Several studies have shown that the MTHFR C677T polymorphism is associated with elevated levels of circulating homocysteine. However, this specific association in postmenopausal women remains controversial. The aim of this meta-analysis was to investigate the association between MTHFR C677T polymorphism and osteoporotic fracture risk in postmenopausal women by conducting a meta-analysis from all eligible case-control studies published to date. 


\section{MATERIAL AND METHODS}

\section{Selection of studies}

The Google Scholar, PubMed, Cochrane Library and China National Knowledge Infrastructure database were searched for all articles on the association between C677T polymorphism and osteoporotic fracture risk in postmenopausal women without language restrictions (1990.1-2013.03), using the following key words: 'MTHFR', 'C677T', 'osteoporotic fracture', 'postmenopausal women', and 'gene polymorphism'. The reference lists of major textbooks, reviews, and included articles were identified through manual searches to find other potentially eligible studies.

\section{Selection criteria}

Studies were included in this meta-analysis if they met the following criteria: i) case-control studies that addressed osteoporotic fracture patients and healthy controls; ii) studies that evaluated the association between C677T polymorphism and osteoporotic fracture risk in postmenopausal women; iii) genotype frequencies of healthy controls were in Hardy-Weinberg equilibrium (HWE). Studies were excluded when: i) not case-control studies that evaluated the association between C677T polymorphism and osteoporotic fracture risk in postmenopausal women; ii) case reports, letters, reviews, and editorial articles; iii) studies that were based on incomplete raw data and no usable data reported; iv) duplicate data were contained in the studies; v) healthy controls were not in HWE.

\section{Data extraction}

Using a standardized form, data from published studies were extracted independently by two reviewers (J.Z.G. and J.S.Z.) to acquire the necessary information. From each of the included articles, the following information was retrieved: first author, year of publication, area, ethnicity, number of cases and controls, genotypes for cases and controls, and evidence of HWE in controls. For conflicting evaluations, an agreement was reached following a discussion.

\section{Statistical analysis}

Meta-analysis was performed using the STATA package version 12.0 (Stata Corporation, College Station, TX, USA). We assessed HWE in the controls for each study using a chi-square test, and $\mathrm{P}<0.05$ was considered to be a significant disequilibrium. The strength of the associations between C677T polymorphism and susceptibility to osteoporotic fracture were estimated by odds ratio (OR) and $95 \%$ confidence interval $(95 \% \mathrm{CI})$ under a homozygote comparison (TT $v_{s} \mathrm{CC}$ ), a heterozygote comparison (TT $v_{s} \mathrm{TC}$ ), a dominant model (CC+TC vs TT), and a recessive mode (TT+TC vs CC) between groups. We quantified the effect of heterogeneity by the I 2 test. I 2 ranges between 0 and $100 \%$ and represents the proportion of inter-study variability that can be attributed to heterogeneity rather than chance. I 2 values of 25,50 and $75 \%$ were defined as low, moderate and high estimates, respectively. When I $>50 \%$ indicated heterogeneity across studies, the random effects model was used for meta-analysis, or the fixed effects model was used. Subgroup analysis was performed according to ethnicity. The different ethnicities were categorized as Asians and Caucasians. Begg's 
funnel plot was used to assess publication bias $(\mathrm{P}<0.05$ was considered to be statistically significant). Sensitivity analysis was performed by altering the statistic models to ensure the stability of measuring results. All P values were two-sided.

\section{RESULTS}

\section{Characteristics of studies}

A total of 282 articles were achieved by literature search from Google Scholar, PubMed, Cochrane Library, and China National Knowledge Infrastructure database, using different combinations of key terms. As shown in Figure 1, 12 eligible studies were retrieved for detailed evaluation. We excluded five studies (one without full-text, one without sufficient genotype data for extraction, 2 meta-analyses and one review). Finally, a total of 7 case-control studies met our inclusion criteria (Jorgensen et al., 2002; Abrahamsen et al., 2003; Li et al., 2004; Bathum et al., 2004; Golbahar et al., 2004; Hong et al., 2007; Yazdanpanah et al., 2008), including 4258 cases and 3454 controls. Table 1 lists the characteristics of each study. There were four studies of Europeans and three of Asians. The genotype distributions in the controls of all studies were in agreement with Hardy-Weinberg equilibrium. The information from these 7 studies and the numbers of cases and controls with TT, TC and CC, genotypes reported in each study are all presented in Table 1.

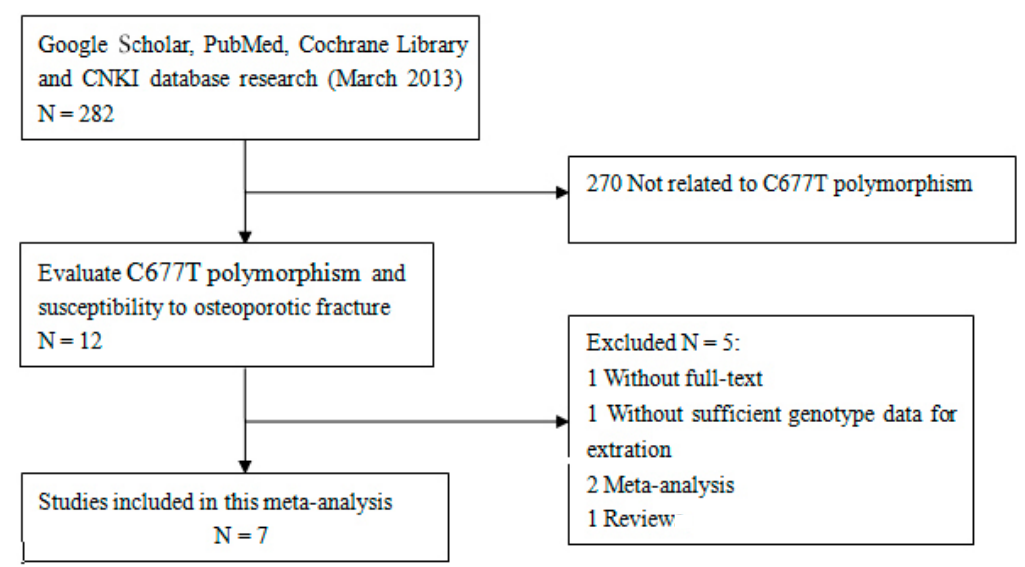

Figure 1. Flow diagram of study searching and selection process.

\section{Table 1. Characteristics of the studies included for meta-analysis.}

\begin{tabular}{|c|c|c|c|c|c|c|c|c|c|c|c|}
\hline \multirow[t]{2}{*}{ Study included } & \multirow[t]{2}{*}{ Year } & \multirow[t]{2}{*}{ Area } & \multirow[t]{2}{*}{ Race } & \multirow[t]{2}{*}{ Cases/Controls } & \multicolumn{3}{|c|}{ Genotypes for cases } & \multicolumn{3}{|c|}{ Genotypes for controls } & \multirow[t]{2}{*}{ HWE test } \\
\hline & & & & & $\mathrm{TT}$ & $\mathrm{TC}$ & $\mathrm{CC}$ & $\mathrm{TT}$ & $\mathrm{TC}$ & $\mathrm{CC}$ & \\
\hline Jorgensen et al. & 2002 & Denmark & Caucasian & $115 / 258$ & 2 & 46 & 67 & 25 & 123 & 110 & 0.26 \\
\hline Abrahamsen et al. & 2003 & Denmark & Caucasian & $473 / 869$ & 43 & 205 & 225 & 74 & 359 & 436 & 0.99 \\
\hline Li et al. & 2004 & China & Asian & $161 / 86$ & 8 & 53 & 100 & 5 & 32 & 49 & 0.94 \\
\hline Hong et al. & 2007 & China & Asian & $883 / 893$ & 121 & 423 & 339 & 131 & 457 & 305 & 0.06 \\
\hline Yazdanpanah et al. & 2008 & Netherlands & Caucasian & $2390 / 552$ & 249 & 1064 & 1077 & 64 & 247 & 241 & 0.95 \\
\hline Agueda et al. & 2010 & Spain & Caucasian & $144 / 627$ & 28 & 66 & 50 & 92 & 314 & 221 & 0.25 \\
\hline Ning et al. & 2012 & China & Asian & $92 / 169$ & 21 & 38 & 33 & 19 & 78 & 72 & 0.76 \\
\hline
\end{tabular}




\section{Results of meta-analysis}

The results of the overall meta-analysis did not suggest any associations between MTHFR C677T polymorphism and osteoporotic fracture susceptibility in postmenopausal women for all genetic models (Table 2 and Figure 2: for TT $v_{S} \mathrm{CC}: \mathrm{OR}=0.99,95 \% \mathrm{CI}=0.72-1.39$, Pheterpgeneity $=0.01$; for TT $v s \mathrm{TC}: \mathrm{OR}=1.02,95 \% \mathrm{CI}=0.87-1.20$, Pheterpgeneity $=0.07$; for CC+TC $v s \mathrm{TT}$ : $\mathrm{OR}=0.96,95 \% \mathrm{CI}=0.71-1.28$, Pheterpgeneity $=0.02$; for $\mathrm{TT}+\mathrm{TC} v s \mathrm{CC}: \mathrm{OR}=0.93,95 \% \mathrm{CI}=$ $0.84-1.03$, Pheterpgeneity $=0.06$ ). When stratified according to ethnicity, we detected no significant association in Caucasians (for TT vs CC: $\mathrm{OR}=0.91,95 \% \mathrm{CI}=0.58-1.45$, Pheterpgeneity $=0.02$; for TT vs TC: $\mathrm{OR}=0.98,95 \% \mathrm{CI}=0.67-1.42$, Pheterpgeneity $=0.08$; for $\mathrm{CC}+\mathrm{TC} v s$ TT: $\mathrm{OR}=0.90$, $95 \% \mathrm{CI}=0.57-1.42$, Pheterpgeneity $=0.00$; for $\mathrm{TT}+\mathrm{TC}$ vs $\mathrm{CC}: \mathrm{OR}=0.91,95 \% \mathrm{CI}=0.72-1.16$, Pheterpgeneity $=0.04$ ) and in Asians (for TT $v_{s} \mathrm{CC}: \mathrm{OR}=1.16,95 \% \mathrm{CI}=0.55-2.44$, Pheterpgeneity $=0.03$; for TT vs TC: $\mathrm{OR}=1.27,95 \% \mathrm{CI}=0.73-2.23$, Pheterpgeneity $=0.12$; for $\mathrm{CC}+\mathrm{TC} v s \mathrm{TT}$ : OR $=0.81,95 \% \mathrm{CI}=0.42-1.57$, Pheterpgeneity $=0.04$; for TT $+\mathrm{TC}$ vs $\mathrm{CC}: \mathrm{OR}=0.87,95 \% \mathrm{CI}=0.74-$ 1.04 , Pheterpgeneity $=0.25$ ). Sensitivity analyses were conducted by altering the statistic models. No material alteration was detected, indicating that our results were statistically robust.

Study
ID

Figure 2. Meta-analysis of the relationship between the MTHFR C677T polymorphism and osteoporotic fracture in postmenopausal women risk for TT $v s$ CC in the race-related subgroup analysis. 
Table 2. Summary of odds ratios (ORs) and 95\% confidence interval $(95 \% \mathrm{CI})$ of MTHFR C677T polymorphism and osteoporotic fracture in postmenopausal women risk.

\begin{tabular}{|c|c|c|c|c|c|c|c|c|c|c|}
\hline \multirow[t]{2}{*}{ Subgroup } & \multirow[t]{2}{*}{ Genetic model } & \multicolumn{2}{|c|}{ Sample size } & \multirow[t]{2}{*}{ Type of model } & \multicolumn{2}{|c|}{ Test of heterogeneity } & \multicolumn{2}{|c|}{ Test of association } & \multicolumn{2}{|c|}{ Test of publication bias } \\
\hline & & Case & Control & & $\mathrm{I}^{2}$ & $\mathrm{P}$ & OR & $95 \% \mathrm{CI}$ & $\mathrm{z}$ & $\mathrm{P}$ \\
\hline \multirow[t]{4}{*}{ Overall } & $\mathrm{TT} v s \mathrm{CC}$ & 4258 & 3454 & Random & $63.9 \%$ & 0.01 & 0.99 & $0.72-1.39$ & 0.30 & 0.76 \\
\hline & $\mathrm{TT} v s \mathrm{TC}$ & & & Fixed & $47.3 \%$ & 0.07 & 1.02 & $0.87-1.20$ & 0.30 & 0.76 \\
\hline & Dominant model & & & Random & $60.3 \%$ & 0.02 & 0.96 & $0.71-1.28$ & 0.30 & 0.76 \\
\hline & Recessive model & & & Fixed & $49.8 \%$ & 0.06 & 0.93 & $0.84-1.03$ & 0.30 & 0.76 \\
\hline \multirow[t]{4}{*}{ Caucasians } & $\mathrm{TT} v s \mathrm{CC}$ & 3122 & 2306 & Random & $69.2 \%$ & 0.02 & 0.91 & $0.58-1.45$ & 0.34 & 1.00 \\
\hline & $\mathrm{TT} v s \mathrm{TC}$ & & & Random & $55.6 \%$ & 0.08 & 0.98 & $0.67-1.42$ & 0.34 & 1.00 \\
\hline & Dominant model & & & Random & $75.6 \%$ & 0.00 & 0.90 & $0.57-1.42$ & 0.24 & 0.81 \\
\hline & Recessive model & & & Random & $64.7 \%$ & 0.04 & 0.91 & $0.72-1.16$ & 0.24 & 0.81 \\
\hline \multirow[t]{4}{*}{ Asians } & $\mathrm{TT} v s \mathrm{CC}$ & 1136 & 1148 & Random & $71.1 \%$ & 0.03 & 1.16 & $0.55-2.44$ & 0.00 & 1.00 \\
\hline & $\mathrm{TT} v s \mathrm{TC}$ & & & Random & $53.1 \%$ & 0.12 & 1.27 & $0.73-2.23$ & 0.00 & 1.00 \\
\hline & Dominant model & & & Random & $68.2 \%$ & 0.04 & 0.81 & $0.42-1.57$ & 0.00 & 1.00 \\
\hline & Recessive model & & & Fixed & $27.8 \%$ & 0.25 & 0.87 & $0.74-1.04$ & 0.00 & 1.00 \\
\hline \multirow{4}{*}{$\begin{array}{l}\text { Sample size } \\
>300\end{array}$} & $\mathrm{TT} v s \mathrm{CC}$ & 4005 & 3199 & Random & $61.4 \%$ & 0.04 & 0.91 & $0.67-1.25$ & & 0.81 \\
\hline & $\mathrm{TT} v s \mathrm{TC}$ & & & Fixed & $40.7 \%$ & 0.15 & 0.98 & $0.82-1.16$ & 0.24 & 0.81 \\
\hline & Dominant model & & & Random & $55.0 \%$ & 0.06 & 1.04 & $0.79-1.36$ & 0.24 & 0.81 \\
\hline & Recessive model & & & Random & $59.4 \%$ & 0.04 & 0.90 & $0.75-1.08$ & 0.24 & 0.81 \\
\hline
\end{tabular}

\section{Publication bias}

Publication bias of the literature was assessed by Begg's funnel plot (Figure 3). The funnel plot was used to measure the asymmetry of the funnel plot. The results of the Begg's funnel plot test are shown in Table 2. Results showed that there was no publication bias (all $\mathrm{P}>0.05$ ).

Begg's funnel plot with pseudo $95 \%$ confidence limits

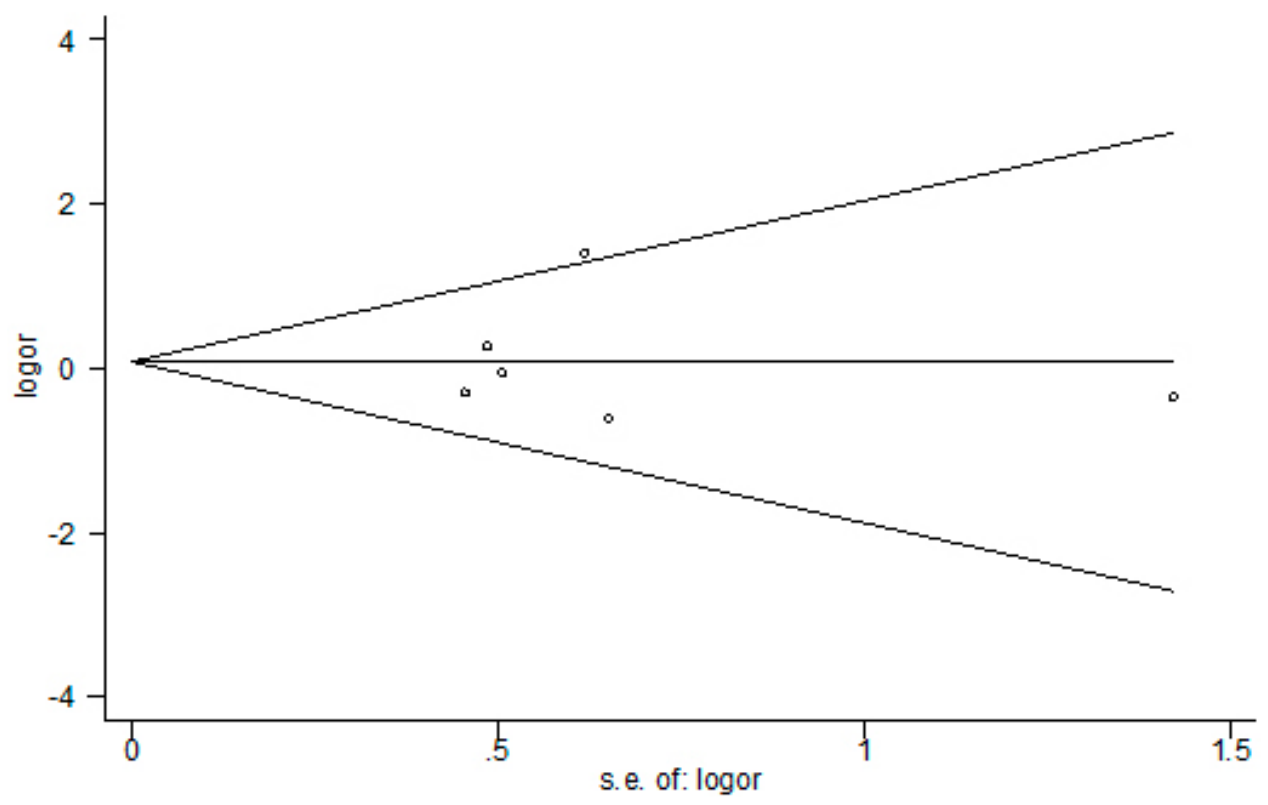

Figure 3. Begg's funnel plot test of publication bias for the association the MTHFR C677T polymorphism and osteoporotic fracture in postmenopausal women risk for TT vs CC. 


\section{DISCUSSION}

An increased homocysteine level appears to be a strong and independent risk factor for osteoporotic fractures (van Meurs et al., 2004). MTHFR plays a key role in the metabolism of homocysteine, and a polymorphism in the MTHFR gene (C677T) is associated with reduced MTHFR activity and increased homocysteine levels. Some studies have reported that C677T polymorphism is associated with the risk of osteoporotic fracture, and other studies have failed to confirm this association. However, the conclusion above based on a small number of samples, suggests that the observed associations between the C677T polymorphism and increased osteoporotic fracture risk reflect chance observations rather than true associations. To help clarify the inconsistent findings, Valero et al. (2007) performed a meta-analysis and found that the MTHFR C677T polymorphism does not appear to be associated with the overall risk of osteoporotic fracture. Nevertheless, postmenopausal women have higher homocysteine levels than premenopausal women (Hak et al., 2000), and several research studies have suggested that the C677T polymorphism is associated with osteoporotic fracture risk in postmenopausal women. Therefore, we performed a meta-analysis aiming at postmenopausal women.

In the current meta-analysis, we examined the association between MTHFR C677T polymorphism and the risk of osteoporotic fracture by critically including all published studies. Finally, only 7 case-control studies were included and comprised a total of 4258 patients and 3454 healthy controls. The main meta-analysis results showed that there was no significant association between MTHFR C677T polymorphism and osteoporotic fracture risk. Considering the result may be affected by ethnicity, we performed a race-related subgroup analysis, and no significant association was found as well between C677T polymorphism and susceptibility to osteoporotic fracture in both Caucasian and Asian populations. When stratifying by sample size $(>300)$, this meta-analysis detected no significant association, suggesting there was no small-study bias in our meta-analysis. There was no evidence of publication bias in this metaanalysis (all $\mathrm{P}>0.05$ ). As the eligible study number was small in this meta-analysis of C677T polymorphism, these results still need further confirmation.

The potential influence of MTHFR C677T polymorphism may be affected via genegene and gene-environment interactions. The effect of C677T polymorphism on fracture could be due to linkage disequilibrium with the lysyl hydroylase 1 (LH1) gene. Mutation in the LH gene is weakened crosslinks in collagen and increased the risk of osteoporotic fracture (Yeowell and Walker, 2000). In addition, plasma concentrations of folate, vitamins B6 and B12 are negatively correlated with plasma homocysteine levels. Increased dietary intake of these vitamins can reduce plasma homocysteine levels (de Bree et al., 2004). Therefore, further studies of the interactions of $\mathrm{C} 677 \mathrm{~T}$ polymorphism and these vitamins (folate, vitamins $\mathrm{B} 6$, and B12) should be taken into consideration to investigate the possible relationships.

There were also some limitations in our meta-analysis. First, because of incomplete raw data or publication limitations, some relevant studies could not be included in our analysis. Second, our systematic review was based on unadjusted data, as the genotype information stratified for the main confounding variables was not available in the original papers and the confounding factors addressed across the different studies were variable. Finally, gene-gene and gene-environment interactions were not tested in the present study, due to the lack of information from the original studies.

In conclusion, our meta-analysis suggests no association between C677T polymor- 
phism and osteoporotic fracture risk, both in Caucasian and Asian populations. Since few studies are available in this field and because current evidence remains limited, large studies with an adequate methodological quality, properly controlling for possible confounds to obtain valid results, are greatly needed.

\section{Conflicts of interest}

The authors declare no conflict of interest.

\section{REFERENCES}

Abrahamsen B, Madsen JS, Tofteng CL, Stilgren L, et al. (2003). A common methylenetetrahydrofolate reductase (C677T) polymorphism is associated with low bone mineral density and increased fracture incidence after menopause: longitudinal data from the Danish osteoporosis prevention study. J. Bone Miner. Res. 18: 723-729.

Agueda L, Urreizti R, Bustamante M, Jurado S, et al. (2010). Analysis of three functional polymorphisms in relation to osteoporosis phenotypes: replication in a Spanish cohort. Calcif. Tissue Int. 87: 14-24.

Bathum L, von Bornemann HJ, Christiansen L, Madsen JS, et al. (2004). Evidence for an association of methylene tetrahydrofolate reductase polymorphism C677T and an increased risk of fractures: results from a population-based Danish twin study. Osteoporos. Int. 15: 659-664.

de Bree A, Mennen LI, Hercberg S and Galan P (2004). Evidence for a protective (synergistic?) effect of B-vitamins and omega-3 fatty acids on cardiovascular diseases. Eur. J. Clin. Nutr. 58: 732-744.

Frosst P, Blom HJ, Milos R, Goyette P, et al. (1995). A candidate genetic risk factor for vascular disease: a common mutation in methylenetetrahydrofolate reductase. Nat. Genet. 10: 111-113.

Golbahar J, Hamidi A, Aminzadeh MA and Omrani GR (2004). Association of plasma folate, plasma total homocysteine, but not methylenetetrahydrofolate reductase C667T polymorphism, with bone mineral density in postmenopausal Iranian women: a cross-sectional study. Bone 35: 760-765.

Hak AE, Polderman KH, Westendorp IC, Jakobs C, et al. (2000). Increased plasma homocysteine after menopause. Atherosclerosis 149: 163-168.

Hong X, Hsu YH, Terwedow H, Tang G, et al. (2007). Association of the methylenetetrahydrofolate reductase C677T polymorphism and fracture risk in Chinese postmenopausal women. Bone 40: 737-742.

Jackson SH (1973). The reaction of homocysteine with aldehyde: an explanation of the collagen defects in homocystinuria. Clin. Chim. Acta 45: 215-217.

Jorgensen HL, Madsen JS, Madsen B, Saleh MM, et al. (2002). Association of a common allelic polymorphism (C677T) in the methylene tetrahydrofolate reductase gene with a reduced risk of osteoporotic fractures. A case control study in Danish postmenopausal women. Calcif. Tissue Int. 71: 386-392.

Li M, Lau EM and Woo J (2004). Methylenetetrahydrofolate reductase polymorphism (MTHFR C677T) and bone mineral density in Chinese men and women. Bone 35: 1369-1374.

Lubec B, Fang-Kircher S, Lubec T, Blom HJ, et al. (1996). Evidence for McKusick's hypothesis of deficient collagen cross-linking in patients with homocystinuria. Biochim. Biophys. Acta 1315: 159-162.

McLean RR, Jacques PF, Selhub J, Tucker KL, et al. (2004). Homocysteine as a predictive factor for hip fracture in older persons. N. Engl. J. Med. 350: 2042-2049.

Melton LJ (1997). Epidemiology of spinal osteoporosis. Spine 22: 2S-11S.

Michaelsson K, Melhus H, Ferm H, Ahlbom A, et al. (2005). Genetic liability to fractures in the elderly. Arch. Intern. Med. 165: 1825-1830.

Ning WQ, Xia J, Wu J, Shen C, et al. (2012). Association of methylenetetrahydrofolate reductase MTHFR polymorphism with osteoporosis in Suzhou postmenopausal women. Acta Univ. Med. Nanjing 32: 1070-1074.

Pongchaiyakul C, Nguyen ND, Jones G, Center JR, et al. (2005). Asymptomatic vertebral deformity as a major risk factor for subsequent fractures and mortality: a long-term prospective study. J. Bone Miner. Res. 20: 1349-1355.

Valero C, Alonso MA, Zarrabeitia MT, Viadero C, et al. (2007). MTHFR C677T polymorphism and osteoporotic fractures. Horm. Metab. Res. 39: 543-547.

van Meurs JB, Dhonukshe-Rutten RA, Pluijm SM, van der Klift M, et al. (2004). Homocysteine levels and the risk of osteoporotic fracture. N. Engl. J. Med. 350: 2033-2041.

Yazdanpanah N, Uitterlinden AG, Zillikens MC, Jhamai M, et al. (2008). Low dietary riboflavin but not folate predicts 
increased fracture risk in postmenopausal women homozygous for the MTHFR $677 \mathrm{~T}$ allele. J. Bone Miner. Res. 23: 86-94.

Yeowell HN and Walker LC (2000). Mutations in the lysyl hydroxylase 1 gene that result in enzyme deficiency and the clinical phenotype of Ehlers-Danlos syndrome type VI. Mol. Genet. Metab. 71: 212-224. 